\title{
Filtered thermal contrast - error analysis
}

by S. Grys* and W. Minkina*

* Faculty of Electrical Engineering, Częstochowa University of Technology, 17 Armii Krajowej Avenue, PL 42-200 Czestochowa,Poland,grys@el.pcz.czest.pl, minkina@el.pcz.czest.pl

\begin{abstract}
A new kind of thermal contrast, called "filtered contrast" is presented, which allows detecting and characterizing material defects using active thermography under some assumptions on physical and thermal parameters of materials. In opposition to known definitions of the thermal contrast, knowledge about defect-free area is not necessary and this contrast is less sensitive to nonuniformity of heat disposal to the material surface. The measurements were performed on an experimental setup equipped with a ThermaCAM PM 595 infrared camera and frame grabber. The step heating was chosen as heat excitation. The results demonstrate usefulness of the 1-D model of heat transfer used for determination of depth of subsurface defects. The influence of the parameter of the smoothing filter, required for filtered contrast implementation, thermal parameters of the tested material and defect on expanded uncertainty of determination of defect depth is also presented. Due to significant complexity of the model of heat transfer the conditions for the "law of propagation of uncertainty" were not fulfilled and a numerical method, i.e. Monte Carlo simulation is applied for the propagation of distributions.
\end{abstract}

\section{Introduction}

Detection of heterogeneity in temperature distribution of thermograms, with no justification in the structure and construction, is a prerequisite to qualify object as defective. Knowledge about defect geometry and depth facilitates looking for cause of defect occurrence. The lack of standards makes thermographic NDT difficult to widespread in industry. This indicates that it is crucial to investigate its metrological properties and limitations for the sake of applications. The occurrence of defects can be caused by:

- disarrangement of the micro- and possibly macrostructure of the object due to the failure of its production process,

- damage due to routine operation.

The most important in object assessment is detection of flat surface and subsurface discontinuities, as most of the critical compressive stresses occur near the surface. For this reason, quick attenuation of thermal waves, which is known disadvantage of TNDT in most cases, does not disqualify this method as a non-destructive method. Typical types of discontinuity are: cracks, delamination, inclusions (solid, metallic and non-metallic with sharp shape, laps) and less severe sub-surface blowholes. The paper deals with detection of defects inside the homogeneous structure of material and determination of depth at which they are located.

\section{Experimental setup}

This section describes the basic features of the experimental setup, located in Czestochowa University of Technology, to study the metrological characteristics of thermogram processing algorithms in active infrared thermography as a method of nondestructive testing. The elements of setup, shown in figure 1, are as follows: a sample of tested material 1 , source of heat excitation $-2,3$ and thermographic system to record the temperature fields on the sample surface $-4,5,6$. Data from an infrared camera (FLIR ThermaCAM PM595), through the external serial interface module -6 and an expansion card IC2 Dig 16 mounted in a PC computer (so-called frame-grabber) are visualized in real time on the PC. The image sequence can be recorded up to 50 frames per second. The aforesaid elements are kept in the ambient temperature and placed in a closed test chamber, which isolates them from external radiation. The radiation emitted inside the chamber, and potentially reflected by its internal walls, is absorbed by high emissivity black paint covering the walls of the chamber. The FLIR ThermaCAM Researcher Professional 2.9 software cooperating with the camera and the frame-grabber was used. The application can show IR images, record them on disk and analyse them afterwards in replay mode. It can provide measurement result values directly from the live stream of images too, but only for the images decided not to record. The measurements are made with the following analysis tools: isotherm, spotmeter, area and line. The results produced by these tools can be displayed within the IR image, in the profile, histogram, table or plot window. Data export to the Matlab *.mat format let to perform the sophisticated data analysis, e.g. smoothing, contrast computation, thermogram segmentation and estimation of defect depth. Single or double lamps can be used as heat excitation. The flash lamps give an impulse and the infrared radiator or the incandescent lamps make it possible to apply a step heating. The double excitation gives better 
uniformity of heat distribution on sample surface than the single one. In the case of the step heating method, it is possible to observe both phases: heating and cooling (long impulse).



Fig. 1. Experimental setup: 1 - sample of tested material, 2 - infrared radiator, 3 - flash lamp with integrated incandescent lamp, 4 - infrared ThermaCAM PM595 camera, 5 - camera holder, 6 - serial interface connected to the frame-grabber

For research purposes a special sample of Plexiglas was made with dimensions shown in figure 2. The face surface of defects was milled, so they have got a cylindrical shape. The Plexiglas is the first layer and the air inside the holes is the second layer of the two-layer sample. To increase the emissivity of the sample surface it was painted with high emissivity black mat paint. The 9 bottom-holes simulate defects in the tested material. One of defect-free areas, called the sound area, is marked as „10" in figure 2.

a)

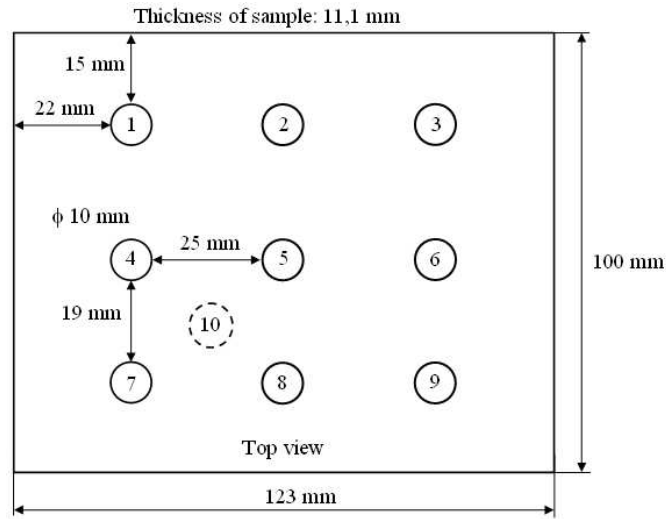

b)



Fig. 2. a) Geometry of tested sample, where: 1-9-defects, 10 - sound area, b) $X Y$ coordinates

The view from the side of the sample is shown in figure 3 with visible exemplary 3 defects, marked $L_{1}, L_{4}$, and $L_{7}$. If we set the origin of $Z$ axis on top surface of sample then thickness of first layer in relation to defects is equivalent to the term defect depth presented in table 1.

a)



b)



Fig. 3. a) Diagram of a two-layer specimen (not to scale), where: $L$ - thickness of first layer, b) ZY coordinates 
Table 1. Defects characteristic

\begin{tabular}{|l|l|l|}
\hline $\begin{array}{l}\text { Defect } \\
\text { No. }\end{array}$ & $\begin{array}{l}\text { Defect depth, } \\
\mathrm{mm}\end{array}$ & $\begin{array}{l}\text { Defect radius } \\
\text { /defect depth }\end{array}$ \\
\hline 1 & 0,8 & 6,2 \\
\hline 2 & 1,0 & 5,0 \\
\hline 3 & 1,1 & 4,5 \\
\hline 4 & 1,3 & 3,8 \\
\hline 5 & 1,7 & 2,9 \\
\hline 6 & 2,0 & 2,5 \\
\hline 9 & 2,1 & 2,4 \\
\hline 7 & 2,3 & 2,2 \\
\hline 8 & 2,5 & 2,0 \\
\hline
\end{tabular}

Table 2. Physical and thermal parameters of tested material and defects

\begin{tabular}{|l|l|l|}
\hline Parameter & Plexiglas & Air (as defect) \\
\hline$\rho, \mathrm{kg} / \mathrm{m}^{3}$ (density) & 1200 & 1,2 \\
\hline$k, \mathrm{~W} /(\mathrm{m} \cdot \mathrm{K})$ (heat conductivity) & 0,2 & 0,02 \\
\hline$a, \mathrm{~m}^{2} / \mathrm{s}$ (thermal diffusivity) & $0,25 \cdot 10^{-6}$ & $33 \cdot 10^{-6}$ \\
\hline$c_{p}, \mathrm{~J} /(\mathrm{kg} \cdot \mathrm{K})$ (specific heat) & 667 & 700 \\
\hline$e, \mathrm{~J} /\left(\mathrm{cm}^{2} \cdot \mathrm{K} \cdot \mathrm{s}^{1 / 2}\right)$ (effusivity) & 0,04 & $4,1 \cdot 10^{-4}$ \\
\hline
\end{tabular}

All defects satisfy the known condition, i.e. the ratio of the radius and depth of the defect is greater than two. Fulfilling this requirement is a preliminary condition of defect detectability using active infrared thermography. The second desired condition is high difference between thermal effusivity of tested material and defects. In the case of arrangement of Plexiglas and air, a thermal mismatch factor $\Gamma$ (in some papers called "reflection ratio") is close to limiting value -1 :

$$
\Gamma_{\text {plexiglas / air }}=\frac{e_{\text {air }}-e_{\text {plex }}}{e_{\text {air }}+e_{\text {plex }}}=-0,977 \approx-1
$$

Its value indicates a good ability of distinguishing between a defect and defect-free areas by analysing the temperature on sample surface. The negative sign indicates that a defect (air) is insulating material in relation to the sample material (Plexiglas). The values of material parameters used for calculation of thermal effusivity $e_{a i r}$ and $e_{\text {plex }}$, presented in table 2 , were taken from reference book [1].

\section{Experiment results}

In the experiment the incandescent lamps integrated with flash lamps were used, each with the power of $250 \mathrm{~W}$. They were placed on both sides of the sample along the $X$ axis, ensuring symmetrical (the two lamps turned on) or asymmetrical (only the right lamp turned on) irradiation of the sample top surface. The reference thermogram at ambient temperature was recorded before lamps are permanently switched on. In next stage, the series of thermograms was recorded up to 120 seconds. Every 2 seconds the thermogram was captured and saved for further analysis performed in offline mode. The data from cooling phase was not used. Exemplary thermograms for symmetrical and asymmetrical heat disposal (two or one incandescent lamps used) are shown in figure 4. Although the sample surface is flat and additionally covered by black matt paint with high emissivity, deformation of the temperature field occurs, caused by heterogeneous surface irradiation even if two lamps symmetrically placed were used. Temperature over hidden defects is affected by local irradiation intensity. Generally, this factor could result in erroneous values of estimated defect depths and needs some special treatment for the sake of quantitative analysis.

a) $15 \mathrm{sec}$



c) $15 \mathrm{sec}$



Fig. 4. Raw thermograms for: $a-b)$ symmetric excitation (left and right lamp), $c$-d) for asymmetric excitation (only right lamp) 


\subsection{Filtered contrast}

In paper [2] a new kind of contrast was proposed, the so-called filtered contrast. Filtered contrast (abbr. FC) is calculated as a subtraction of raw thermogram and smoothed thermogram. Eq. (1) describes principle of operation of FC for particular pixel indexed as $(x, y)$ :

$$
F C_{x, y}=T_{x, y}-\operatorname{filter}\left(T_{x, y}\right)
$$

FC has the same unit as temperature and FC is zero for defect-free areas. Possible material's defects appear above the constant background level. The "filter $\left(T_{x, y}\right)$ " routine can be performed in many manners, e.g. polynomial approximation, morphological opening operation or two-dimensional Gaussian filtering. Choosing the last idea, the smoothing is applied to raw thermal images along the columns and rows according to the following weighting function:

$$
f(i)=\exp \left(-\frac{1}{2} \cdot\left(\frac{i-\frac{i_{\max }}{2}}{B}\right)^{2}\right)
$$

for $i=<1, i_{\max }>$ where $i_{\max }=7 \cdot B+1$. Function $f(I)$ is symmetric with maximum in the middle of the range of $i$, i.e. for $i=i m a x / 2$. To avoid undesirable amplification of gain, some extra data scaling operations must be performed. Parameter $B$ is arbitrarily chosen at this stage of our research. As a result of filtration and subtraction, the background adjustment (quasi equal temperature for all pixels for defect-free areas) is visible in figures 5 . It facilitates thermal image segmentation into two classes: "defect" and "no defect". Comparing the raw thermograms recorded after 15 seconds from switching on the heat excitation, i.e. figure $4 \mathrm{a}$ and $4 \mathrm{c}$ with figure $5 \mathrm{a}$ and $5 \mathrm{c}$, it can be noted that the use of filtered contrast allowed an early detection of more than one of defects - figure $4 \mathrm{c}$ and figure $5 \mathrm{c}$.
a) $15 \mathrm{sec}$
b) $50 \mathrm{sec}$





c) $15 \mathrm{sec}$ d) $50 \mathrm{sec}$



Fig. 5. Filtered contrast FC for: $a-b)$ symmetric excitation (left and right lamp), c-d) for asymmetric excitation (only right lamp)

So far, only temperature gradients on a single thermogram were analysed. To estimate depth of detected defects the range of temperature changes and their rate in time must be inspected with help of mathematical model of heat transfer. According to [3], the temperature increase above initial temperature $T_{o}$ on the surface, due to the step heating, is given by equation:

$$
\Delta T_{x, y}(\tau)=C_{x, y} \sqrt{\tau}\left\{1+\sum_{n=1}^{\infty} 2(-\Gamma)^{n}\left[\exp \left(-\frac{n^{2} L_{x, y}{ }^{2}}{a_{\text {plex }} \cdot \tau}\right)-\frac{n L_{x, y} \sqrt{\pi}}{\sqrt{a_{\text {plex }} \tau}} \operatorname{erf}\left(\frac{n L_{x, y}}{\sqrt{a_{\text {plex }} \tau}}\right)\right]\right\}=C_{x, y} \sqrt{\tau}\left[1+f\left(L_{x, y}, \Gamma, \tau, a_{p l e x}\right)\right]
$$

where

$$
C_{x, y}=f\left(\varepsilon_{x, y}, R_{x, y}, I_{x, y}, e_{p l e x}\right)
$$

is a constant term related to the energy of absorption, $\tau$ - time, $\varepsilon_{x, y}$ - surface emissivity, $R_{x, y}-$ reflectivity of sample surface, $I_{x, y}$ - radiation intensity, $\mathrm{W} / \mathrm{m}^{2}$. The $(x, y)$ are coordinates of the midpoint of detected defects. In the general case $C_{x, y}$ can take 
different values for each pixel indexed by $(x, y)$, mainly due to nonuniformity of radiation intensity - figure 4 . Hence, the temperature of the midpoint of a defect seen by infrared camera is related to the defect depth and unfortunately is affected by local radiation intensity. When looking at figure 6 , it can be observed the lack of compliance of order of experimental curves with the order of the defect depth listed in table 1. For example, defect 9 seems to lie deeper than defect 7 . If single lamp is used, the situation is even worse. Defect 3 appears to lie much deeper than 4 and the same is true for defects 6 and 7 .

a)



b)



Fig. 6. Filtered contrast FC as a function of square root of time for the midpoints of areas 1-10: a) symmetric excitation (left and right lamp), b) for asymmetric excitation (only right lamp)

If we assume following relative formula:

$$
\frac{C_{x, y} \sqrt{\tau}\left[1+f\left(L_{x, y}, \Gamma, \tau, a_{\text {plex }}\right)\right]-C_{x, y} \sqrt{\tau}\left[1+f\left(L, \Gamma, \tau, a_{\text {plex }}\right)\right]}{C_{x, y} \sqrt{\tau}\left[1+f\left(L, \Gamma, \tau, a_{\text {plex }}\right)\right]}=\frac{f\left(L_{x, y}, \Gamma, \tau, a_{\text {plex }}\right)-f\left(L, \Gamma, \tau, a_{\text {plex }}\right)}{1+f\left(L, \Gamma, \tau, a_{\text {plex }}\right)}
$$

then on the right side of Eq. (6) factor $C_{x, y}$ no longer occurs. Function $f(L, \Gamma, \tau, a)$ is called "normalized temperature" [1]. It is dimensionless, hence the right side of Eq. (6) is also dimensionless. This property is present also in a new kind of contrast, called relative filtered contrast RFC, proposed in [2]:

$$
R F C_{x, y}=\frac{T_{x, y}-\text { filter }\left(T_{x, y}\right)}{\text { filter }\left(T_{x, y}\right)-T_{o}}
$$

where $T_{o}$ is a reference temperature of sample surface before step heating was started. The value of RFC does not depend on scale of temperature so it can be used in comparative studies. In practice the sample of material is often in ambient temperature before heat source is turned on. Taking a thermogram when temperature of object is close to the ambient temperature is ill-conditioned [4]. Instead of considering the $T_{0}$ for characteristic pixel $(x, y)$ for each defect, the arithmetic mean of temperature of region of interest of reference thermogram should be chosen. It means that only one constant value of $T_{0}$ is applied to all defects.

Application of Eq. (4) requires a finite number of components of the sum. Simulation shows that for more than 10 components of the sum the changes in the shape of curves of temperature increases are negligible.

The procedure for quantitative assessment can be performed as follows:

1. record a reference thermogram before heating,

2. evaluate the $T_{o}$ as an arithmetic mean of the temperatures of reference thermogram,

3. start heat excitation (step heating),

4. record a series of thermograms,

5. assume the value of the parameter $B$ of $2 D$ Gaussian filter,

6. apply for all thermograms the relative filtered contrast RFC according to Eq. (7),

7. locate the defects manually or automatically on the basis of significant temperature in relation to the homogeneous background using any binarization method, e.g. [5],

8. determine the $(x, y)$ coordinates of characteristic points of detected defects, e.g. the midpoint of circle shaped defects,

9. calculate the value of mismatch factor $\Gamma$ for sample material and defect or assume it if calculation is not possible,

10. fit the temperature curves from the experiment processed with RFC to these obtained from model given by right side of Eq. (6) for characteristic points with coordinates $(x, y)$,

11. estimate defect depth using e.g. least squares method. 


\section{Error and uncertainty analysis}

Every new promising data processing technique requires examination of its properties. It precedes successful transfer to the industrial practice. Error or uncertainty analysis is an important stage of validation process. Error of determination of defect depth located at $(x, y)$ coordinates depends on, inter alia: parameter $B$ of Gaussian smoothing routine, reference temperature $T_{0}, L_{x, y} / L$ ratio, mismatch factor $\Gamma$, diffusivity of sample material (here Plexiglas), the time of data registration (i.e. number of thermograms) and 1D model limitations. Evaluation of the $\Gamma$ value according to the Eq. (1) requires both the effusivity of sample material and defect. Effusivity can be simply expressed by following equation:

$$
e=\sqrt{k \cdot \rho \cdot c_{p}}
$$

The real values of parameters occurring in above equation may significantly vary from those published in various handbooks or, in general case, there is no assumption about material of defect. If we take approximated values of effusivity of Plexiglas and air with some errors, they only slightly affect the value of mismatch factor $\Gamma$. For example, if we assume relative error of evaluation of effusivity $\delta e_{p l e x}= \pm 50 \%$ and $\delta e_{a i r}= \pm 50 \%$ and approximated values (taken from handbook) of effusivity are $e_{\text {plex }}=0,04 \mathrm{~J} /\left(\mathrm{cm}^{2} \cdot \mathrm{K} \cdot \mathrm{s}^{-1 / 2}\right)$ and $e_{\text {air }}=0,00041 \mathrm{~J} /\left(\mathrm{cm}^{2} \cdot \mathrm{K} \cdot \mathrm{s}^{-1 / 2}\right)$ then depending on the combination of errors' signs, the relative error $\delta \Gamma$ will be as presented in table 3 .

Table 3. Relative error of mismatch factor $\Gamma$

\begin{tabular}{|l|l|l|l|l|}
\hline & $\delta e_{\text {air }}=50 \%$ & $\delta e_{\text {air }}=-50 \%$ & $\delta e_{\text {air }}=50 \%$ & $\delta e_{\text {air }}=-50 \%$ \\
& $\delta e_{\text {plex }}=50 \%$ & $\delta e_{\text {plex }}=50 \%$ & $\delta e_{\text {plex }}=-50 \%$ & $\delta e_{\text {plex }}=-50 \%$ \\
\hline$\delta \Gamma$ & 0 & $-1,36$ & 4,19 & 0 \\
\hline
\end{tabular}

Calculations were carried out on the basis of classical definition of errors [4]. Approximated value of mismatch factor was calculated according to the Eq. (1). True values of effusivity of Plexiglas and air are:

$$
e_{\text {air_tRUE }}=\frac{e_{\text {air }}}{\delta e_{\text {air }} / 100 \%+1} \quad \text { and } \quad e_{\text {plex } \_ \text {TRUE }}=\frac{e_{\text {plex }}}{\delta e_{\text {plex }} / 100 \%+1} . \text { (8) }
$$

True value of mismatch factor is:

$$
\Gamma_{\text {plex } / \text { air_tRUE }}=\frac{e_{\text {air_tRUE }}-e_{\text {plex_tRUE }}}{e_{\text {air_tRUE }}+e_{\text {plex_tRUE }}}
$$

and needed relative error of mismatch factor is:

$$
\delta \Gamma_{\text {plex } / \text { air }}=\frac{\Gamma_{\text {plex } / \text { air }}-\Gamma_{\text {plex } / \text { air_tRUE }}}{\Gamma_{\text {plex } / \text { air_tRUE }}} 100 \%
$$

Table 3 shows that for assumed level of errors the relative error $\delta \Gamma$ does not exceed $5 \%$ in the worst case. This is caused by significant difference between thermal and physical parameters of Plexiglas and air. In general case, for other arrangement of materials, this error could be larger.

The analytical analysis of influence of all indicated in this section input quantities on the uncertainty of determination of defect depth is a very sophisticated task. Let's consider a relatively simple problem. For example if a complex parameter $L_{x, y} / \sqrt{a_{\text {plex }}}$ is estimated from Eq. (4) and $a_{p l e x}$ is approximated with limiting absolute error $\Delta a_{p l e x}$, hence passing over the influence of other quantities, the question is: what absolute error of $\Delta L_{x, y}$ is? Due to significant complexity of the method of determination of defect depth (nonlinear model of heat transfer, Gaussian filtering, least squares minimalization), the conditions for „law of propagation of uncertainty” are not fulfilled and a numerical method for the propagation of distributions must be applied for error analysis. In ref. [6], treated as supplement to Guide to the Expression of Uncertainty in Measurement [7], an interesting procedure is proposed. It gives recommendation how the uncertainty could be evaluated in case of complexity of the model. The procedure applies to evaluation of $95 \%$ coverage interval for the output quantity value. The described procedure consists of the following stages [6]: 
a) define the output quantity, the quantity required to be measured,

b) decide the input quantities upon which the output quantity depends,

c) develop a model relating the output quantity to these input quantities,

d) on the basis of available knowledge assign probability density functions to the values of the input quantities,

e) propagate the probability density functions of the input quantities through the model to obtain the probability density function for the output quantity value obtain from it:

1) its expectation, taken as the estimate of the output quantity value,

2) its standard deviation, taken as the standard uncertainty associated with the estimate of the output quantity value,

3 ) the coverage interval containing the unknown output quantity value with a specified probability.

Due to limited volume of this paper the results of uncertainty analysis are presented only for worse case, i.e. lamps placed asymmetrically (only right lamp). Similar results were obtained for a second case, i.e. lamps placed symmetrically. In this study, the mismatch factor $\Gamma$ and the parameter a (diffusivity) of Plexiglas were assumed to have influence on the output quantities, i.e. defects depth. The parameter $B$ of smoothing filter was arbitrary chosen from 6 to 16 to ensure fulfilment of both qualitative and quantitative objectives. The number of thermograms and error of reference temperature $T_{0}$ was not investigated. To perform aimed analysis, a uniform symmetric distribution of probability of input quantities was assumed with interval $\Gamma=[-1,-0,9]$ for Plexiglas-air arrangement what correspond to the range of limiting errors of effusivity of both materials - table 3. Diffusivity of Plexiglas is considered from a range $a=[0,225 ; 0,275] \cdot 10^{-6} \mathrm{~m}^{2} / \mathrm{s}$ what correspond to $\pm 10 \%$ of relative error of diffusivity of Plexiglas taken from [1]. To obtain the distribution function of output quantity the Monte Carlo simulation technique was applied. For each defect 1-9 the depth was computed and estimates of distribution function were carried out. According to point $\mathrm{e}$ ) of the above mentioned procedure, an expectation was obtained and presented in table 4 . The closest value of expectation to true value of depth was additionally marked. A significant number of these cases occur for $B$ from 9 10. $95 \%$ coverage intervals are presented in table 5 and the narrowest ones are extra marked. A significant number of these cases occur for $B$ from 9-14. Table 6 says if $95 \%$ coverage interval contains the true value of depth. A significant number of these cases occurred for $B$ from 9-12.

Table 4. Estimate of the defect depth as a function of parameter $B$



Table 5. 95\% coverage interval as a function of parameter $B$

\begin{tabular}{|c|c|c|c|c|c|c|c|c|c|}
\hline \multirow{3}{*}{$B$} & \multicolumn{9}{|c|}{$95 \%$ coverage interval, $\mathrm{mm}$} \\
\hline & \multicolumn{9}{|c|}{ Defect No.: } \\
\hline & 1 & 2 & 3 & 4 & 5 & 6 & 9 & 7 & 8 \\
\hline 6 & {$[1,00 ; 1,23]$} & {$[1,19 ; 1,44]$} & {$[1,42 ; 1,68]$} & {$[1,61 ; 1,88]$} & {$[2,04 ; 2,34]$} & {$[2,41 ; 2,74]$} & {$[2,70 ; 3,06]$} & {$[2,41 ; 2,75]$} & {$[2,82 ; 3,20]$} \\
\hline 7 & {$[0,88 ; 1,10]$} & {$[1,07 ; 1,31]$} & {$[1,27 ; 1,52]$} & {$[1,45 ; 1,71]$} & {$[1,84 ; 2,12]$} & {$[2,18 ; 2,49]$} & {$[2,40 ; 2,73]$} & {$[2,20 ; 2,51]$} & {$[2,55 ; 2,89]$} \\
\hline 8 & {$[0,78 ; 0,99]$} & {$[0,97 ; 1,19]$} & {$[1,16 ; 1,40]$} & {$[1,32 ; 1,57]$} & {$[1,71 ; 1,98]$} & {$[2,05 ; 2,34]$} & {$[2,26 ; 2,58]$} & {$[2,04 ; 2,34]$} & {$[2,42 ; 2,75]$} \\
\hline 9 & {$[0,72 ; 0,93]$} & {$[0,92 ; 1,14]$} & {$[1,09 ; 1,32]$} & {$[1,25 ; 1,48]$} & {$[1,61 ; 1,87]$} & {$[1,93 ; 2,21]$} & {$[2,11 ; 2,40]$} & {$[1,93 ; 2,22]$} & {$[2,27 ; 2,59]$} \\
\hline 10 & {$[0,66 ; 0,88]$} & {$[0,86 ; 1,08]$} & {$[1,03 ; 1,26]$} & {$[1,17 ; 1,41]$} & {$[1,53 ; 1,80]$} & {$[1,86 ; 2,14]$} & {$[2,04 ; 2,34]$} & {$[1,84 ; 2,13]$} & {$[2,21 ; 2,52]$} \\
\hline 11 & {$[0,63 ; 0,84]$} & {$[0,83 ; 1,05]$} & {$[0,98 ; 1,21]$} & {$[1,12 ; 1,36]$} & {$[1,47 ; 1,73]$} & {$[1,78 ; 2,05]$} & {$[1,93 ; 2,21]$} & {$[1,76 ; 2,04]$} & {$[2,10 ; 2,40]$} \\
\hline 12 & {$[0,60 ; 0,81]$} & {$[0,80 ; 1,02]$} & {$[0,95 ; 1,18]$} & {$[1,08 ; 1,31]$} & {$[1,43 ; 1,69]$} & {$[1,75 ; 2,02]$} & {$[1,91 ; 2,20]$} & {$[1,71 ; 1,99]$} & {$[2,08 ; 2,39]$} \\
\hline 13 & {$[0,59 ; 0,80]$} & {$[0,79 ; 1,00]$} & {$[0,93 ; 1,15]$} & {$[1,05 ; 1,28]$} & {$[1,40 ; 1,65]$} & {$[1,70 ; 1,97]$} & {$[1,84 ; 2,12]$} & {$[1,66 ; 1,94]$} & {$[2,01 ; 2,31]$} \\
\hline 14 & {$[0,56 ; 0,77]$} & {$[0,76 ; 0,98]$} & {$[0,90 ; 1,12]$} & {$[1,01 ; 1,24]$} & {$[1,36 ; 1,61]$} & {$[1,67 ; 1,94]$} & {$[1,83 ; 2,11]$} & {$[1,62 ; 1,89]$} & {$[1,99 ; 2,29]$} \\
\hline 15 & {$[0,56 ; 0,76]$} & {$[0,76 ; 0,97]$} & {$[0,89 ; 1,11]$} & {$[1,00 ; 1,23]$} & {$[1,34 ; 1,59]$} & {$[1,64 ; 1,90]$} & {$[1,78 ; 2,05]$} & {$[1,58 ; 1,85]$} & {$[1,94 ; 2,23]$} \\
\hline 16 & {$[0,54 ; 0,74]$} & {$[0,74 ; 0,95]$} & {$[0,88 ; 1,09]$} & {$[0,97 ; 1,19]$} & {$[1,32 ; 1,56]$} & {$[1,63 ; 1,89]$} & {$[1,78 ; 2,05]$} & {$[1,56 ; 1,81]$} & {$[1,94 ; 2,22]$} \\
\hline
\end{tabular}


Table 6. Validation of the method of defect depth determination

\begin{tabular}{|c|c|c|c|c|c|c|c|c|c|}
\hline \multirow{3}{*}{$B$} & \multicolumn{9}{|c|}{$\begin{array}{l}\text { Is true value of defect depth lying inside the } \\
95 \% \text { coverage interval?, } \mathrm{Y} / \mathrm{N}\end{array}$} \\
\hline & \multicolumn{9}{|c|}{ Defect No.: } \\
\hline & 1 & 2 & 3 & 4 & 5 & 6 & 9 & 7 & 8 \\
\hline 6 & $\mathrm{~N}$ & $\mathrm{~N}$ & $\mathrm{~N}$ & $\bar{N}$ & $\mathrm{~N}$ & $\mathrm{~N}$ & $\mathrm{~N}$ & $\mathrm{~N}$ & $\mathrm{~N}$ \\
\hline 7 & $\mathrm{~N}$ & $\mathrm{~N}$ & $\mathrm{~N}$ & $\mathrm{~N}$ & $\mathrm{~N}$ & $\mathrm{~N}$ & $\mathrm{~N}$ & $Y$ & $\mathrm{~N}$ \\
\hline 8 & $Y$ & $Y$ & $\mathrm{~N}$ & $\mathrm{~N}$ & $\mathrm{~N}$ & $\mathrm{~N}$ & $\mathrm{~N}$ & $Y$ & $Y$ \\
\hline 9 & $\bar{Y}$ & $Y$ & $Y$ & $\bar{Y}$ & $Y$ & $\mathrm{Y}$ & $\mathrm{N}$ & $\mathrm{N}$ & $Y$ \\
\hline 10 & $\bar{Y}$ & $Y$ & $\bar{Y}$ & $\bar{Y}$ & $Y$ & $Y$ & $\bar{Y}$ & $\mathrm{~N}$ & $Y$ \\
\hline 11 & $\bar{Y}$ & $Y$ & $Y$ & $\bar{Y}$ & $Y$ & $Y$ & $Y$ & $\mathrm{~N}$ & $\mathrm{~N}$ \\
\hline 12 & $\bar{Y}$ & $Y$ & $Y$ & $Y$ & $\mathrm{~N}$ & $Y$ & $Y$ & $\mathrm{~N}$ & $\mathrm{~N}$ \\
\hline 13 & $\mathrm{~N}$ & $Y$ & $Y$ & $\mathrm{~N}$ & $\mathrm{~N}$ & $\mathrm{~N}$ & $Y$ & $\mathrm{~N}$ & $\mathrm{~N}$ \\
\hline 14 & $\mathrm{~N}$ & $\mathrm{~N}$ & $Y$ & $\mathrm{~N}$ & $\mathrm{~N}$ & $\mathrm{~N}$ & $Y$ & $\mathrm{~N}$ & $\mathrm{~N}$ \\
\hline 15 & $\mathrm{~N}$ & $\mathrm{~N}$ & $Y$ & $\mathrm{~N}$ & $\mathrm{~N}$ & $\mathrm{~N}$ & $\mathrm{~N}$ & $\mathrm{~N}$ & $\mathrm{~N}$ \\
\hline 16 & $\mathrm{~N}$ & $\mathrm{~N}$ & $\mathrm{~N}$ & $\mathrm{~N}$ & $\mathrm{~N}$ & $\mathrm{~N}$ & $\mathrm{~N}$ & $\mathrm{~N}$ & $\mathrm{~N}$ \\
\hline
\end{tabular}

As many as 8 of 9 of defects were properly characterized for $B=10$ with the exception of defect no. 7 . The relative accuracy of depth estimation, calculated as a larger value of relative difference between the limits of $95 \%$ confidence interval and the true value of defect depth is presented in table 7 for $B=10$.

Table 7. Accuracy of the method of defect depth determination, \%

\begin{tabular}{|c|c|c|c|c|c|c|c|c|}
\hline \multicolumn{2}{|l}{ Defect No.: } \\
1 & 2 & 3 & 4 & 5 & 6 & 9 & 7 & 8 \\
\hline-17 & -14 & -15 & -10 & -10 & \pm 7 & 11 & -20 & -12 \\
\hline
\end{tabular}

\section{Summary}

The method of defect depth estimation requires an assumption about parameter $B$, whose value strongly affects the accuracy of this estimation. The optimal value of $B$ corresponds to the diameter of defects. The larger diameter of the defect, the greater value of $B$ is required to properly smooth a thermogram hiding a defect against the background of defect-free sub-areas. This aspect will be examined in further work. For the inspected sample, assumed diameter of defects, $B=10$ and assumed accuracy of estimation of diffusivity of Plexiglas the accuracy of the method does not exceed $20 \%$ even for deep defects. To fully validate the described method an exhaustive study must be carried out in the next stage of research, but obtained results are optimistic so far.

\section{REFERENCES}

[1] Maldague X., "Theory and practice of infrared technology for nondestructive testing," John Wiley \& Sons, Inc., New York, 2001.

[2] Grys S., Minkina W. and Chudzik S., "Detekcja wad podpowierzchniowych na podstawie analizy serii termogramów (eng. Detection of subsurface defects on the basis of thermogram analysis)," Proceedings of XVII Symposium on Modelowanie i Symulacja Systemow Pomiarowych (eng. Modelling and Simulation of Measuring Systems), Krynica Gorska (Poland), p. 2735 - in polish.

[3] Murphy J.C., Aamodt L.C., Maclachlan Spicer J.W., "Principles of photothermal detection in solids" in Mandelis A, ed. "Principles and perspectives of photothermal and photoacoustic phenomena," Elsevier, (New York), 1992, p. 41-94.

[4] Minkina W. and Dudzik S., "Infrared thermography - errors and uncertainties," John Wiley \& Sons Ltd, Chichester, 2009.

[5] Otsu N., "A threshold selection method from gray-level histograms," IEEE Transactions on Systems. Man and Cybernetics, 1979, vol. 9, no. 1, p. 62-66.

[6] "Guide to the expression of uncertainty in measurement. Supplement 1. Numerical methods for the propagation of distributions," Joint Committee for Guides in Metrology, 2004.

[7] "Evaluation of measurement data - Guide to the expression of uncertainty in measurement," [GUM 1995 with minor corrections], JCGM, (100) 2008. 